\title{
Prognostic value of a modified surprise question designed for use in the emergency department setting
}

\author{
Samir A. Haydar', Tania D. Strout ${ }^{1}$, Alicia G. Bond ${ }^{2}$, Paul KJ Han ${ }^{3}$ \\ 'Department of Emergency Medicine, Maine Medical Center, Tufts University School of Medicine, Portland, \\ ME, USA \\ ${ }^{2}$ Department of Emergency Medicine, Providence Medford Medical Center, Medford, OR, USA \\ ${ }^{3}$ Center for Outcomes Research \& Evaluation, Maine Medical Center, Tufts University School of Medicine, \\ Portland, ME, USA
}

Objective Few reliable and valid prognostic tools are available to help emergency physicians identify patients who might benefit from early palliative approaches. We sought to determine if responses to a modified version of the surprise question, "Would you be surprised if this patient died in the next 30 days" could predict in-hospital mortality and resource utilization for hospitalized emergency department patients.

Methods For this observational study, emergency physicians responded to the modified surprise question with each admission over a five-month study period. Logistic regression analyses were completed and standard test characteristics evaluated.

Results 6,122 visits were evaluated. Emergency physicians responded negatively to the modified surprise question in 918 (15.1\%). Test characteristics for in-hospital mortality were: sensitivity $32 \%$, snecificity $85 \%$, positive predictive value $6 \%$, negative predictive value $98 \%$. The risk of intensi'ie care unit use (relative risk [RR], 1.87; 95\% confidence interval [CI], 1.45 to 2.40), use of 'comfort measures' orders ( $\mathrm{RR}, 3.43 ; 95 \% \mathrm{Cl}, 2.81$ to 4.18), palliative-care consultation ( $\mathrm{RR}, 3.06$; $95 \% \mathrm{Cl} 2.62$ to 3.56$)$, and in-hospital mortality ( $\mathrm{RR}, 2.18 ; 95 \% \mathrm{Cl}, 1.72$ to 2.76$)$ were greater for patients with negative responses.

Conclusion The modified surprise question is a simple trigger for palliative care needs, accurately identifying those at greater risk for in-hospital mortality and resource utilization. With a negative predictive value of $98 \%$, affirmative responses to the modified surprise question provide reassurar!ce that in-hospital death is unlikely.

Keywords Palliative medicine; Terminal care; Emergency service, hospital; Surprise question
elSSN: 2383-4625

Received: 23 December 2017

Revised: 13 March 2018

Accepted: 6 April 2018

Correspondence to: Tania D. Strout Department of Emergency Medicine, Maine Medical Center, Tufts University School of Medicine, 22 Bramhall Street, Portland, ME 04102, USA

E-mail: strout@mmc.org ORCID

http://orcid.org/0000-0001-9053-1523

An abstract describing this work was presented at the 2015 Society for Academic Emergency Medicine annual meeting, Dallas, TX, USA.

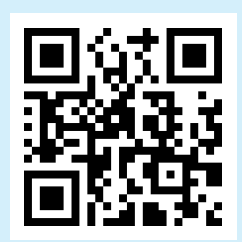

How to cite this article:

Haydar SA, Strout TD, Bond AG, Han PKJ. Prognostic value of a modified surprise question designed for use in the emergency department setting. Clin Exp Emerg Med 2019;6(1):70-76.
This is an Open Access article distributed under the terms of the Creative Commons Attribution Non-Commercial License (http:// creativecommons.org/licenses/by-nc/4.0/). 


Capsule
Summary is already known
$\begin{aligned} & \text { Attention is turning to the role of emergency physicians in identifying patients with unmet palliative care needs. Few } \\ & \text { reliable and valid prognostic tools are available to identify those who might benefit from an early palliative approach. }\end{aligned}$
$\begin{aligned} & \text { What is new in the current study } \\ & \text { The modified version of the surprise question serves as a simple trigger for palliative care needs assessment in the acute } \\ & \text { care setting, accurately identifying those at greater risk for in-hospital mortality and resource utilization. With a nega- } \\ & \text { tive predictive value of 98\%, an affirmative response to the modified version of the surprise question provides reassur- } \\ & \text { ance that in-hospital death is unlikely. However, a negative response holds promise as an effective mechanism to iden- } \\ & \text { tify patients who might benefit from implementation of an early palliative approach. }\end{aligned}$

\section{INTRODUCTION}

A growing body of literature suggests that many patients visiting the emergency department (ED) have unmet palliative care (PC) needs, and that identifying these patients early in the course of disease will extend and improve quality of life, reduce hospitalizations and future ED visits. ${ }^{1-4}$ As interest in improving $P C$ in the ED grows, development of widely-applicable screening tools for PC needs is a priority. While there has been significant work on the assessment of PC needs in cancer patients visiting the ED, ${ }^{1,5}$ there are currently a paucity screening tools validated in the general ED population.

Dozens of "triggers" for PC needs assessments have been described in the literature. Such triggers are often based on diagnosis or symptoms, healthcare utilization patterns, psychosocial needs, and/or the clinical impressions of providers. ${ }^{3,6}$ We believe an ideal initial screening tool or trigger for the fast-paced ED environment would be easy to administer in a brief period of time; easy to understand and interpret; require minimal training to apply; and would be specific enough that further assessment on patients screening positive would be feasible. A trigger based on clinical impression may be particularly well-suited to ED physicians, who regularly employ intuitive pattern-recognition techniques to make rapid clinical judgments.

The surprise question (SO), "Would you be surprised if this patient died in the next year?" has been used as a PC screening tool in cancer, dialysis, and general populations to predict one-year mortality with moderate sensitivity and high specificity. ${ }^{5,7-10}$ Unfortunately these studies were small with limited generalizability and notably high mortality rates in the populations studied. While the simplicity of this trigger makes it appealing for the ED environment, the one-year time frame is likely to have limited clinical utility and be conceptually challenging for the busy emergency physician. In an attempt to improve the clinical relevance of the
SO, while at the same time maintain its simplicity, here we proposed a modified ED version of the surprise question (ED-MSO), "Would you be surprised if this patient died in the next 30 days?" for use in the ED. The ED-MSO is a clinician-based tool that is simple to administer and easy for providers to interpret. If found to be reliable, the ED-MSO could be applied easily in the ED setting. Therefore, our objective was to determine if the ED-MSO, as answered by ED physicians at time of admission, could reliably predict increases in hospital resource utilization, in-hospital mortality and other surrogates for unmet PC needs. We hypothesized that in-hospital mortality, intensive care unit (ICU) use, utilization of a 'comfort measures' order set and PC consultation would be higher in the 'No, I would not be surprised' group as compared to the positive response group (Yes, I would be surprised).

\section{METHODS}

\section{Study design}

We conducted a retrospective review of prospectively collected data to address our research objectives. We developed a mechanism within our electronic health record (EPIC) to prospectively query emergency providers at the time of admission on their response to the ED-MSO. At our institution all patients entering through the ED and subsequently admitted to hospital receive emergency provider generated ED transition admitting orders at the time of the admission decision. During this intervention, embedded within the ED transition order set was the ED-MSO with a hard stop forcing providers to answer. As a result, providers were prospectively queried on nearly $100 \%$ of patients admitted through our ED. Patients taken directly to the operating room or cardiac catheterization laboratory bypassed this process. The study period lasted 5 months from August 1, 2013 through December 31, 2013. Emergency physician responses to the modified SO were used to assign patients to one of two groups: those for 
whom physicians would be surprised if death occurred within 30 days (Yes, I would be surprised group) and those for whom physicians would not be surprised if death occurred within 30 days (No, I would not be surprised group). The Maine Medical Center (MMC) institutional review board exempted the study and waived the requirement for written informed consent.

\section{Study setting}

The study was conducted at MMC, a 637-bed tertiary care teaching hospital located in northern New England. The ED at MMC is a mixed adult and pediatric ED that treats approximately 65,000 patients annually and houses an emergency medicine residency training program.

At the time of this intervention, significant institutional efforts were focused on improving end-of-life care. The ED-MSO intervention, as described here, was only part of a greater awareness leading to an acceleration of educational efforts targeting providers hospital wide.

\section{Selection of the study sample}

Patients were actively enrolled and admitting physicians queried by virtue of admission to the hospital through the ED. We obtained visit-level data through queries of MMC's electronic medical records system (Epic Systems Corporation, Verona, WI, USA). As ED patient care occurs, the Epic system electronically captures time, date, demographic, and clinical data throughout the hospital stay, yielding an integrated electronic medical record. We analyzed de-identified data for all ED visits occurring during the study period spanning August 1, 2013 through December 31, 2013. Data analyzed for this manuscript followed the 'go live' of the ED-MSO functionality in the electronic record by approximately 6 months.

Data obtained included patient demographic information, triage acuity, presenting complaint, patient disposition from the ED, patient disposition from the hospital, inpatient hospitalization unit, utilization of the institution's 'comfort measures' order set, ordering of a PC consult, and emergency physician responses to the ED-MSO. We excluded primary psychiatric visits, visits for patients who were not admitted to the hospital, and visits for patients who were less than 18 years of age and those taken directly to the operating theater or cardiac catheterization laboratory from the ED.

\section{Outcome measures}

The primary outcome measure of interest in this study was inpatient mortality which we defined as any patient death that occurred prior to discharge from the ED or inpatient hospital set- ting. Secondary outcomes of interest included markers of hospital resource utilization; placement of orders for 'comfort measures', PC consultation, ICU utilization and length of stay. We also investigated the association of responses with various patient demographic characteristics (Table 1).

Table 1. Characteristics of the study subjects

\begin{tabular}{|c|c|c|c|}
\hline Characteristics & $\begin{array}{l}\text { All subjects } \\
(n=6,122)\end{array}$ & $\begin{array}{l}\text { No, I would not } \\
\text { be surprised } \\
\quad(n=918)\end{array}$ & $\begin{array}{l}\text { Yes, I would } \\
\text { be surprised } \\
(n=5,171)\end{array}$ \\
\hline \multicolumn{4}{|l|}{ Age category (yr) } \\
\hline $18-29$ & $380(6.2)$ & $11(1.2)$ & $367(7.1)$ \\
\hline $30-39$ & 408 (6.7) & $17(1.9)$ & $390(7.5)$ \\
\hline $40-49$ & $618(10.1)$ & $27(2.9)$ & $586(11.3)$ \\
\hline $50-59$ & 951 (15.5) & $75(8.2)$ & 873 (16.9) \\
\hline $60-69$ & $1,108(18.1)$ & $135(14.7)$ & 963 (18.6) \\
\hline $70-79$ & $1,234(20.2)$ & $222(24.2)$ & $1,009(19.5)$ \\
\hline $80-89$ & 1,065 (17.4) & $298(32.5)$ & 760 (14.7) \\
\hline$\geq 90$ & 358 (5.8) & $133(14.5)$ & $223(4.3)$ \\
\hline \multicolumn{4}{|l|}{ Sex } \\
\hline Male & 3,168 (51.7) & $487(53.1)$ & $2,658(51.4)$ \\
\hline Female & 2,954 (48.3) & $431(46.9)$ & $2,513(48.6)$ \\
\hline \multicolumn{4}{|l|}{ ESI category } \\
\hline ESI-1 & $130(2.2)$ & $50(5.7)$ & $79(1.6)$ \\
\hline ESI-2 & 4,229 (71.2) & $668(75.6)$ & $3,539(70.5)$ \\
\hline ESI-3 & $1,526(25.7)$ & $161(18.2)$ & $1,355(27.0)$ \\
\hline ESI-4 & $51(0.9)$ & $4(0.5)$ & $47(0.9)$ \\
\hline ESI-5 & $1(>0.01)$ & $1(0.1)$ & $0(0)$ \\
\hline \multicolumn{4}{|l|}{ Race } \\
\hline White & 5,852 (95.7) & $30(3.3)$ & $233(4.5)$ \\
\hline Non-White/other & $265(4.3)$ & 888 (96.7) & $4,934(95.5)$ \\
\hline \multicolumn{4}{|l|}{ Ethnicity } \\
\hline Hispanic & $45(0.7)$ & $8(0.9)$ & $36(0.7)$ \\
\hline Non-Hispanic & 6,036 (99.3) & 903 (99.1) & $5,102(99.3)$ \\
\hline \multicolumn{4}{|l|}{ Payor type } \\
\hline Private & $1,495(24.4)$ & 75 (8.2) & $1,412(27.3)$ \\
\hline Public & 4,263 (69.6) & 824 (89.8) & $3,418(66.1)$ \\
\hline Self-pay & 364 (5.9) & $19(2.1)$ & 341 (6.6) \\
\hline \multicolumn{4}{|l|}{ Intensive care use } \\
\hline Yes & $171(2.8)$ & $47(5.2)$ & $124(2.4)$ \\
\hline No & 5,951 (97.2) & 871 (94.9) & $5,047(97.6)$ \\
\hline \multicolumn{4}{|c|}{ Comfort measures orderset use } \\
\hline Yes & $109(1.8)$ & $54(5.9)$ & $55(1.1)$ \\
\hline No & $5,980(98.2)$ & $864(94.1)$ & $5,116(98.9)$ \\
\hline \multicolumn{4}{|c|}{ Palliative care consult order } \\
\hline Yes & $270(4.4)$ & $114(12.4)$ & $156(3.0)$ \\
\hline No & 5,819 (95.6) & $804(87.6)$ & $5,015(97.0)$ \\
\hline \multicolumn{4}{|l|}{ In-hospital mortality } \\
\hline Yes & $157(2.6)$ & $107(11.7)$ & $50(0.9)$ \\
\hline No & $5,932(97.4)$ & $811(88.3)$ & $5,154(99.6)$ \\
\hline
\end{tabular}

Values are presented as number (\%).

ESI, Emergency Severity Index. 


\section{Primary data analysis}

We received the study data in a Microsoft Excel (Microsoft Corporation, Redmond, WA, USA) spreadsheet and later transferred the data into IBM SPSS Statistics ver. 22 (IBM Corp., Armonk, NY, USA) for analysis. To maximize sample size, we excluded cases with missing data on an analysis-by-analysis basis rather than excluding an entire case. Findings for categorical variables are reported as numbers and percentages with 95\% confidence intervals (Cls). Continuous variables are reported as means and $\mathrm{Cls}$ or medians and interquartile ranges, as appropriate.

For our primary analysis, we first conducted univariate logistic regression analyses to examine the relationships between patient characteristics (age, race, ethnicity, sex, acuity level, and payor type), clinical resource utilization (ED length of stay, hospital length of stay, presence of a PC consult order, ICU utilization, and use of the institutional 'comfort measures' orderset) and emergency physician responses to the ED-MSO with in-hospital mortality. Reference groups were chosen by temporal order or increasing values. Factors that were significant in univariate logistic regression analyses were then used as covariates in subsequent multivariate logistic regression modeling. Through multivariate modeling, we have estimated odds ratios and corresponding 95\% Cls.

In addition to these primary analyses, standard test characteristics, including sensitivity, specificity, positive predictive value, and negative predictive value, were also computed. We also calculated the relative risk (RR) of experiencing each outcome (ICU utilization, PC consultation, 'comfort measures' order set use, and inhospital mortality) based upon the physicians' ED-MSO response.

An a priori sample size calculation was not computed; rather, we used the sample available to us approximately six months following implementation of the ED-MSO. Following standard sample size recommendations, the available sample of data from 6,122 cases is adequate to support the analyses we conducted. ${ }^{11,12}$

\section{RESULTS}

\section{Characteristics of the study subjects}

During the study, there were 7,766 admissions to the hospital originating in the ED. We excluded from analysis 768 admissions for patients $<18$ years of age, and 876 primary psychiatric admissions, leaving data from 6,122 cases for analysis. Direct admissions to the hospital and patients taken directly to the operating room and cardiac catheterization laboratory were also excluded as they bypassed our admission process. The median age for those included was 66 years (interquartile range, 51 to 79); 3,168 (52\%) were male and 5,852 (96\%) were white. Emergency physicians responded 'No, I would not be surprised' if the patient died in the next 30 days for 918 cases (15\%). Differences in age, Emergency Severity Index category, payor type, ICU utilization, 'comfort measures' order set use, PC consult ordering, and inhospital mortality were all noted when comparing the characteristics of patients with negative (No, I would not be surprised) and affirmative (Yes, I would be surprised) physician responses (Table 2). In-hospital mortality was $2.6 \%$ during the study period (157 cases), with 107 deaths (68\%) in the 'No, I would not be surprised' group and 50 (32\%) in the 'Yes, I would be surprised' group.

\section{Characteristics of the modified SO}

The sensitivity, specificity, positive predictive value (PPV) and negative predictive value (NPV) for the ED-MSO were computed for all subjects as well as for three subgroups based upon age ( $\geq 60$ vs. 18-59 years old), subject sex, and ICU utilization (Table 2). For the entire cohort, the sensitivity of the ED-MSO negative response (No, I would not be surprised) was 32\% (95\% Cl, 25\% to 40\%) and the specificity was $85 \%(95 \% \mathrm{Cl}, 84 \%$ to $86 \%)$. The PPV was $5.5 \%(95 \% \mathrm{Cl}, 4 \%$ to $7 \%)$ and the NPV was $97.9 \%(95 \% \mathrm{Cl}, 97.5 \%$ to $98.3 \%$ ).

Table 2. Test characteristics for the modified surprise question by provider response for hospital mortality

\begin{tabular}{|c|c|c|c|c|c|c|}
\hline Group & $\begin{array}{l}\text { No. of } \\
\text { patients }\end{array}$ & $\begin{array}{l}\text { No, I would not be } \\
\text { surprised responses }\end{array}$ & Sensitivity ${ }^{a)}$ & Specificity ${ }^{b)}$ & PPV ${ }^{c}$ & $N^{\prime} V^{d)}$ \\
\hline All patients & 6,089 & $918(15.1)$ & $31.8(24.7-39.8)$ & 85.4 (84.4-86.3) & $5.45(4.1-7.1)$ & 97.9 (97.5-98.3) \\
\hline Age $18-59$ years & 2,346 & $130(5.5)$ & $17.1(6.61-33.7)$ & 94.63 (93.6-95.5) & $4.62(1.7-9.8)$ & $98.7(98.1-99.1)$ \\
\hline Age $\geq 60$ years & 3,743 & $788(21.1)$ & $36.1(27.6-45.3)$ & 79.5 (78.1-80.8) & $5.6(4.1-7.4)$ & $97.4(96.7-97.9)$ \\
\hline Female & 2,944 & $431(14.6)$ & $31.9(21.2-44.2)$ & 85.8 (84.4-87.0) & $5.1(3.2-7.6)$ & $98.1(97.5-98.6)$ \\
\hline Male & 3,145 & $487(15.5)$ & $31.8(22.3-42.6)$ & $85.0(83.7-86.2)$ & $5.7(3.9-8.2)$ & 97.7 (97.1-98.3) \\
\hline $\mathrm{ICU}$ & 5,918 & $871(14.7)$ & $33.8(23.0-46.0)$ & 85.5 (84.6-86.4) & $2.8(1.8-4.1)$ & 99.1 (98.8-99.3) \\
\hline Yes & 171 & 47 (27.5) & $30.2(20.8-41.1)$ & $75.3(64.8-84.0)$ & $55.3(40.1-69.8)$ & $51.6(24.5-60.7)$ \\
\hline
\end{tabular}

Values are presented as number (\%) or \% (95\% confidence interval).

PPV, positive predictive value; NPV, negative predictive value.

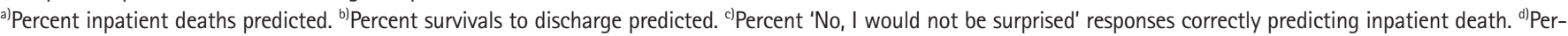
cent "Yes, I would be surprised" responses correctly predicting survival to discharge. 
Table 3. Predictive value of the modified surprise question

\begin{tabular}{|c|c|c|c|c|}
\hline Characteristics & $\begin{array}{l}\text { All subjects } \\
(n=6,122)\end{array}$ & $\begin{array}{l}\text { No, I would not be surprised } \\
\qquad(n=918)\end{array}$ & $\begin{array}{l}\text { Yes, I would be surprised } \\
\qquad(n=5,171)\end{array}$ & $\begin{array}{l}\text { Relative risk } \\
(95 \% \mathrm{Cl})\end{array}$ \\
\hline \multicolumn{5}{|c|}{ Intensive care use } \\
\hline No & 5,918 (97.2) & $871(94.9)$ & $5,047(97.2)$ & \\
\hline \multicolumn{5}{|c|}{ Comfort measures order set use } \\
\hline Yes & 109 (1.8) & $54(5.9)$ & $55(1.1)$ & $3.43(2.81-4.18)$ \\
\hline Yes & $270(4.4)$ & $114(12.4)$ & $156(3.0)$ & $3.06(2.62-3.56)$ \\
\hline No & 5,819 (95.6) & $804(87.6)$ & 5,015 (97.0) & \\
\hline \multicolumn{5}{|c|}{ In-hospital mortality } \\
\hline Yes & $157(2.6)$ & $50(5.4)$ & $107(2.1)$ & $2.18(1.72-2.76)$ \\
\hline No & $5,932(97.4)$ & 868 (94.6) & $5,064(97.9)$ & \\
\hline
\end{tabular}

Values are presented as number (\%).

$\mathrm{Cl}$, confidence interval.

Table 4. Univariate logistic regression analysis to predict in-hospital mortality

\begin{tabular}{lc}
\hline Predictor & OR (95\% Cl) \\
\hline $\begin{array}{l}\text { Modified 'surprise question' response } \\
\text { (reference = 'yes') }\end{array}$ & $0.367(0.260-0.517)$ \\
Age category (yr, reference: 18-29) & \\
$30-39$ & $0.931(0.130-6.643)$ \\
$40-49$ & $3.743(0.833-16.814)$ \\
$50-59$ & $3.853(0.893-16.623)$ \\
$60-69$ & $4.900(1.162-20.667)$ \\
$70-79$ & $5.355(1.281-22.394)$ \\
$80-89$ & $7.376(1.774-30.667)$ \\
$\geq 90$ & $11.183(2.595-48.200)$ \\
Sex (reference: male) & $1.195(0.868-1.644)$ \\
Race (reference: White) & $0.281(0.069-1.141)$ \\
Ethnicity (reference: non-Hispanic) & $0.892(0.122-6.516)$ \\
Payor type (reference: private) & \\
Public & $1.806(1.171-2.784)$ \\
Self-pay & $0.819(0.311-2.154)$ \\
ED length of stay & $0.998(0.997-0.999)$ \\
Hospital length of stay & $1.033(1.017-1.050)$ \\
Intensive care use (reference: no) & $83.791(57.285-122.562)$ \\
Comfort measures order set use (reference: no) & $85.758(55.715-132.001)$ \\
Palliative care consult order (reference: no) & $8.476(5.782-12.424)$ \\
\hline
\end{tabular}

$\mathrm{OR}$, odds ratio; $\mathrm{Cl}$, confidence interval; $\mathrm{ED}$, emergency department.

\section{Predictive value of the modified SO}

We computed the RR of ICU utilization, PC consultation, 'comfort measures' order set utilization, and in-hospital mortality for subjects with and without negative ED-MSO responses (Table 3). Those with negative responses were at greater risk of being treated in the ICU (RR, 1.87; 95\% Cl, 1.45 to 2.40), of receiving PC consultation ( $\mathrm{RR}, 3.06 ; 95 \% \mathrm{Cl}, 2.62$ to 3.56), of 'comfort measures' or-
Table 5. Multivariate logistic regression analysis to predict in-hospital mortality

\begin{tabular}{lcc}
\hline Predictor & OR (95\% Cl) & P-value \\
\hline $\begin{array}{l}\text { Modified surprise question response } \\
\text { (reference: yes) }\end{array}$ & $0.975(0.595-1.600)$ & 0.921 \\
Age $\geq 60$ yr (reference: $<60 \mathrm{yr})$ & $1.848(1.057-3.232)$ & $0.031^{\text {a) }}$ \\
$\begin{array}{l}\text { Payor type (reference: private) } \\
\quad 1.062(0.594-1.901)\end{array}$ & 0.838 \\
$\quad$ Public & $0.621(0.189-2.047)$ & 0.434 \\
$\quad$ Self-pay & $1.000(0.998-1.001)$ & 0.447 \\
ED length of stay & $1.032(1.007-1.057)$ & $0.011^{\text {a) }}$ \\
Hospital length of stay & $104.837(64.160-171.303)$ & $<0.001^{\text {a) }}$ \\
Intensive care use (reference: no) & $78.791(41.533-149.472)$ & $<0.001^{\text {a) }}$ \\
Comfort measures order set use & & \\
$\quad$ (reference: no) & $1.552(0.828-2.910)$ & 0.171 \\
Palliative care consult order (reference: no) & & \\
\hline
\end{tabular}

$\mathrm{OR}$, odds ratio; $\mathrm{Cl}$, confidence interval; $\mathrm{ED}$, emergency department.

a) Statistically significant predictor.

der set use (RR, 3.43; 95\% Cl, 2.81 to 4.18) and of experiencing in-hospital mortality (RR, 2.18; $95 \% \mathrm{Cl}, 1.72$ to 2.76$)$.

\section{In-hospital mortality}

In univariate logistic regression analysis, the modified SO response, patient age, payor type, ED length of stay, hospital length of stay, ICU utilization, 'comfort measures' order set utilization, and PC consult orders were significantly associated with inpatient mortality (Table 4). The odds of experiencing in-hospital death were $63 \%$ (95\% Cl, 48\% to 74\%) less likely for patients where the emergency physician indicated, 'Yes, I would be surprised' when compared to those for whom physicians indicated that the patient's death within 30 days would not surprise them. Patients treated in the ICU died in-hospital 84 times more frequently (95\% 
$\mathrm{Cl}, 57$ to 123) than patients who were not treated in the ICU while those with 'comfort measures' orders died 86 times (95\% Cl, 56 to 132 times) more frequently than patients without 'comfort measures' orders. In multivariate analysis, only age, hospital length of stay, ICU utilization and 'comfort measures' predictors were significantly associated with inpatient mortality (Table 5). The odds of inpatient mortality were 9 times greater $(95 \% \mathrm{Cl}, 6$ to 12) for patients with $P C$ consult orders than for their counterparts without PC consults.

\section{DISCUSSION}

In our cohort a "No" response to the ED-MSO, "Would you be surprised if this patient died in the next 30 days?" appears to offer prognostic value regarding increased resource utilization for admitted patients and appears to help predict inpatient mortality. The risk of in-hospital mortality, use of comfort-only order set, and discharge to hospice-all markers of unmet PC needs at time of ED visit-were all higher for patients in the "No, I would not be surprised" group. Our results demonstrate that the ED-MSO may be used by ED physicians as a simple, effective time-of-admission trigger for a more comprehensive PC needs assessment.

A landmark study published in 2010 by Temel demonstrated that early PC for patients with metastatic lung cancer leads to reduction of symptoms, improved both quality of life and survival despite less-aggressive end-of-life care. ${ }^{13}$ Since that time, a rapidly-expanding evidence base has supported these findings for a broad range of terminal diagnoses. More recently, the first randomized study of ED-triggered PC for patients with advanced cancer reproduced the key results of the Temel study, suggesting attention to unmet PC needs in the ED can help patients to live better and longer lives. ${ }^{1}$

Despite evidence suggesting the benefits of PC are strongest when PC teams are engaged early in the course of terminal disease, $P C$ is still underutilized to be of maximum benefit. ${ }^{5}$ Emergency physicians, who have no long-term relationships with terminally-ill patients but who meet them in moments of crisis, may be uniquely positioned to objectively identify those unmet needs. Furthermore, as a point of access to medical care for patients across the socioeconomic and health literacy spectra, the ED has been identified as a key point for identification of unmet PC needs in vulnerable populations without access to high-quality outpatient care. $^{14}$

The ED-MSO is simple to implement, requires no training to answer, and is easily incorporated into time-of-admission orders in an electronic medical record. Unlike diagnosis-based triggers, which are being used in current studies on ED-triggered PC as- sessments, ${ }^{15}$ the ED-MSO may capture both those with known terminal illness and those with an unexpected change in health who may have a more urgent need for PC assessment. Given its high specificity for in-hospital mortality and hospice discharge, the ED-MSO alone could be used to trigger the use of a more comprehensive assessment tool in a subset of inpatients. Although high sensitivity is generally considered to be an important characteristic of an initial screening tool, most hospitals do not currently have the resources to perform comprehensive PC assessments on the number of patients that would be identified with a highly-sensitive but poorly-specific tool.

The high NPV (97.9\%; 95\% Cl, 97.5\% to 98.3\%) for in-hospital mortality observed in this cohort indicates that when emergency physicians provide an affirmative response to the ED-MSO (yes, I would be surprised), the probability of surviving to hospital discharge is approximately $98 \%$. This may be clinically useful for considering a physician's desire to avoid failing to provide helpful resources to the sickest of patients, those who might die before leaving the hospital, particularly in environments with limited PC resources. Should a physician respond 'yes, I would be surprised', it would be quite unlikely that their patient would expire during the hospitalization. In resource-limited settings without extensive PC resources, the ED-MSO may help to identify those who are least likely to have acute need for early initiation of $\mathrm{PC}$, therefore allowing for resources to be targeted at those who are most in need.

Given the low sensitivity and PPV for in-hospital mortality demonstrated in this population (which does not include cardiac catheterization patients and those taken directly to the operating room), providers should be aware of the severity of illness in their cohort population. We believe the value for this tool for the ED setting is two-fold. First, it provides a simple trigger for emergency clinicians to be thinking about early initiation of PC. In addition, it may provide value in targeting resources towards those most acutely in need.

Our study was limited to a single tertiary-care medical center $E D$, where the particular culture and practice patterns may influence responses to the ED-MSO. Although the ED-MSO was initiated at the time of admission for all patients admitted to the hospital from the ED, patients taken directly to the operating theater or cardiac catheterization laboratory, bypassed this typical admission process and therefore did not have the ED-MSO answered. As this particular population likely represents a disproportionately ill segment of patients entering our institution, had they been included as part of this intervention, it is likely that the prognostic value of the ED-MSO would have been stronger. In addition, the data used in this analysis were de-identified, rendering it impos- 
sible for us to account for multiple visits by the same patient. As a result, each encounter was handled as an independent visit.

It should also be noted that the ED-MSO will likely work best to identify the sickest of patients with PC needs, one would suspect that it may not adequately identify those patients who are not terminally ill but have underlying conditions such as severe COPD, HIV and various malignancies that could also benefit from early PC services. Furthermore, as implemented here, the ED-MSO does not help to identify those patients in need of PC services that are discharged from the ED. While we suspect there are patients discharged from our ED with unmet PC needs the described intervention was not designed to identify this segment of our population. We also acknowledge that in-hospital mortality may not fully represent true 30-day mortality statistics; unfortunately national death records were not available to us at the time of this pilot study.

While the focus of this study was not on identifying patients with PC needs early in their disease process, it was intended to identify these patients earlier in their hospital course. Future research should also focus on mechanism to optimize the identification and management of patients with unmet PC needs earlier in their illness trajectory.

Emergency physicians are uniquely positioned to improve symptom burden and quality of life in patients with terminal illness by identifying unmet PC needs at the time of admission. The ED-MSO is a simple, clinician-based trigger for PC needs assessment that may help to identify patients with a significantly-higher risk of mortality and use of PC resources. We hope to further define the clinical implications of this tool with future investigations targeting the tool's ability to predict outcomes in specific ED patient populations, as well as develop further work flow optimizations that help facilitate PC consultation based on the ED-MSO's responses.

\section{CONFLICT OF INTEREST}

No potential conflict of interest relevant to this article was reported.

\section{REFERENCES}

1. Grudzen $C R$, Richardson LD, Johnson PN, et al. Emergency department-initiated palliative care in advanced cancer: a randomized clinical trial. JAMA Oncol 2016 Jan 14. https://doi. org/10.1001/jamaoncol.2015.5252.

2. Delgado-Guay MO, Kim YJ, Shin SH, et al. Avoidable and un- avoidable visits to the emergency department among patients with advanced cancer receiving outpatient palliative care. J Pain Symptom Manage 2015;49:497-504.

3. George N, Phillips E, Zaurova M, Song C, Lamba S, Grudzen C. Palliative care screening and assessment in the emergency department: a systematic review. J Pain Symptom Manage 2016;51:108-19.

4. Wu FM, Newman JM, Lasher A, Brody AA. Effects of initiating palliative care consultation in the emergency department on inpatient length of stay. J Palliat Med 2013;16:1362-7.

5. Kistler EA, Sean Morrison R, Richardson LD, Ortiz JM, Grudzen CR. Emergency department-triggered palliative care in advanced cancer: proof of concept. Acad Emerg Med 2015;22:237-9.

6. George N, Barrett N, McPeake L, Goett R, Anderson K, Baird J. Content validation of a novel screening tool to identify emergency department patients with significant palliative care needs. Acad Emerg Med 2015;22:823-37.

7. Rhee J, Clayton JM. The 'surprise' question may improve the accuracy of GPs in identifying death in patients with advanced stage IV solid-cell cancer. Evid Based Med 2015;20:71.

8. Hamano J, Morita T, Inoue $S$, et al. Surprise questions for survival prediction in patients with advanced cancer: a multicenter prospective cohort study. Oncologist 2015;20:839-44.

9. Feyi $K$, Klinger $S$, Pharro $G$, et al. Predicting palliative care needs and mortality in end stage renal disease: use of an at-risk register. BMJ Support Palliat Care 2015;5:19-25.

10. O'Callaghan A, Laking G, Frey R, Robinson J, Gott M. Can we predict which hospitalised patients are in their last year of life? A prospective cross-sectional study of the Gold Standards Framework Prognostic Indicator Guidance as a screening tool in the acute hospital setting. Palliat Med 2014;28:1046-52.

11. Green SB. How many subjects does it take to do a regression analysis. Multivariate Behav Res 1991;26:499-510.

12. Cohen J, Cohen P. Applied multiple regression/correlation analysis for the behavioral sciences. Hillsdale, NJ: Erlbaum; 1975.

13. Temel JS, Greer JA, Muzikansky A, et al. Early palliative care for patients with metastatic non-small-cell lung cancer. $\mathrm{N}$ Engl J Med 2010;363:733-42.

14. Knott DA, Hiestand BC. If not us, then who? Palliative care referral from the emergency department. Acad Emerg Med 2015; 22:227-8.

15. Bowman J, George N, Barrett N, Anderson K, Dove-Maguire K, Baird J. Acceptability and reliability of a novel palliative care screening tool among emergency department providers. Acad Emerg Med 2016;23:694-702. 